\title{
FINITE ELEMENT DIFFERENTIAL FORMS ON CUBICAL MESHES
}

\author{
DOUGLAS N. ARNOLD AND GERARD AWANOU
}

\begin{abstract}
We develop a family of finite element spaces of differential forms defined on cubical meshes in any number of dimensions. The family contains elements of all polynomial degrees and all form degrees. In two dimensions, these include the serendipity finite elements and the rectangular BDM elements. In three dimensions they include a recent generalization of the serendipity spaces, and new $H$ (curl) and $H$ (div) finite element spaces. Spaces in the family can be combined to give finite element subcomplexes of the de Rham complex which satisfy the basic hypotheses of the finite element exterior calculus, and hence can be used for stable discretization of a variety of problems. The construction and properties of the spaces are established in a uniform manner using finite element exterior calculus.
\end{abstract}

\section{INTRODUCTION}

In this paper we develop a family of finite element spaces $\mathcal{S}_{r} \Lambda^{k}\left(\mathcal{T}_{h}\right)$ of differential forms, where $\mathcal{T}_{h}$ is a mesh of cubes in $n \geq 1$ dimensions, $r \geq 1$ is the polynomial degree, and $0 \leq k \leq n$ is the form degree. Thus, in 3 dimensions, the space $\mathcal{S}_{r} \Lambda^{k}\left(\mathcal{T}_{n}\right)$ is a finite element subspace of the Hilbert space $H^{1}, H(\operatorname{curl}), H(\mathrm{div})$, or $L^{2}$, according to whether $k=0,1,2$, or 3 . For $n=1$ or 2 , the spaces were previously known, while in three (or more) dimensions, they are mostly new. Specifically, our construction yields a new family of $H$ (curl) elements and a new family of $H$ (div) elements on cubical meshes in three dimensions. Our treatment in an exterior calculus framework allows all the spaces and their properties to be developed together. The spaces combine together in complexes satisfying the basic hypotheses of the finite element exterior calculus [5]. This means that, in addition to their use individually, they can be used in pairs, $\mathcal{S}_{r+1} \Lambda^{k-1}\left(\mathcal{T}_{h}\right) \times \mathcal{S}_{r} \Lambda^{k}\left(\mathcal{T}_{h}\right)$ in a variety of mixed finite element applications, with stability and convergence following from the abstract theory of [5]. Element diagrams for some of the spaces are shown in Figure 1. The dimension of the shape function spaces are given in Theorem 3.6 below and are tabulated for $n \leq 4$ and $r \leq 7$ in Table 1 .

Since the development of the first stable mixed finite elements for the Poisson equation by Raviart and Thomas in 1977, such elements have proven to be powerful tools for numerical computation. Their paper [11] introduces a family of finite element discretizations of the space $H(\operatorname{div}, \Omega)$ for a two-dimensional domain $\Omega$, one

Received by the editor April 11, 2012 and, in revised form, December 21, 2012.

2010 Mathematics Subject Classification. Primary 65N30.

Key words and phrases. Mixed finite elements, finite element differential forms, finite element exterior calculus, cubical meshes, cubes.

The work of the first author was supported in part by NSF grant DMS-1115291.

The work of the second author was supported in part by NSF grant DMS-0811052 and the Sloan Foundation. 
$r=1$
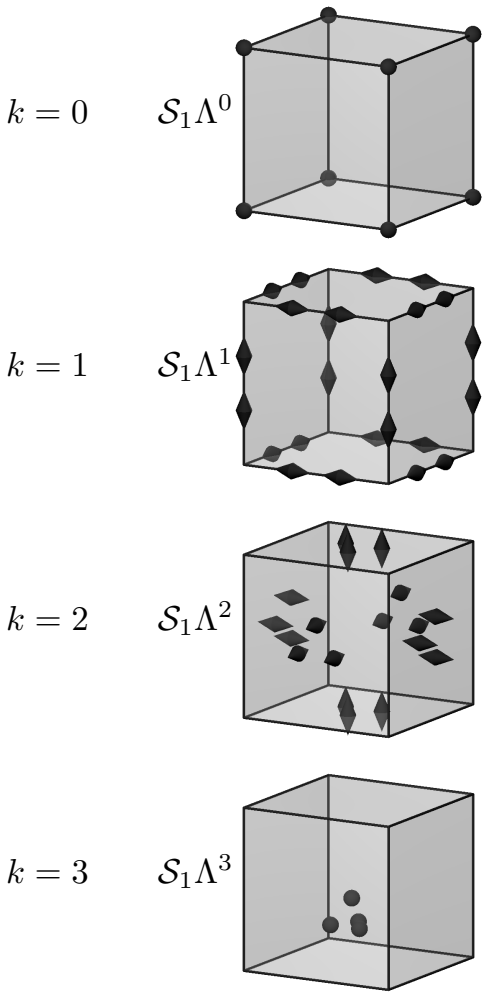

$r=2$
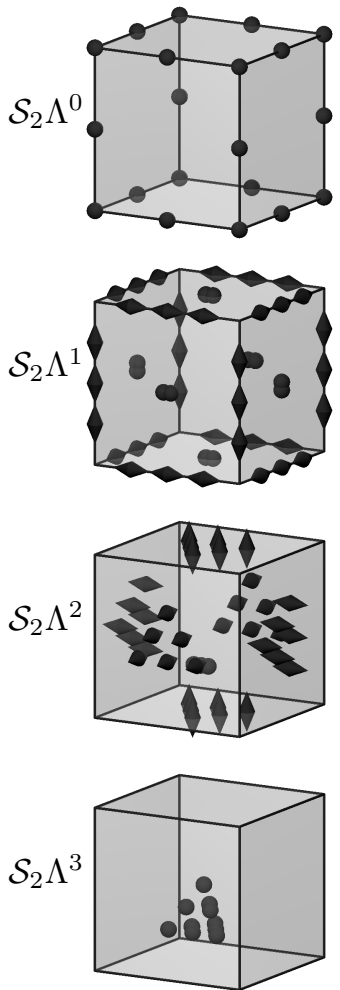

$r=3$
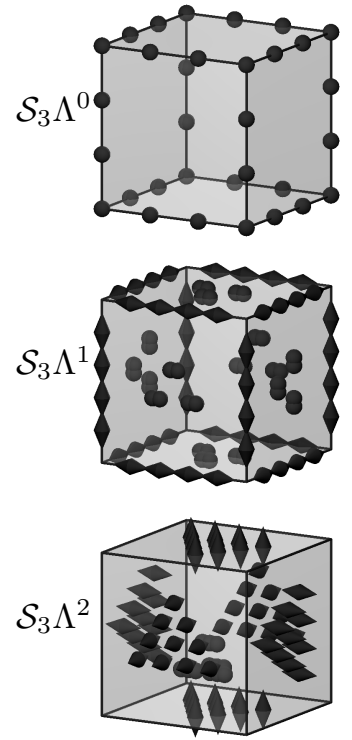

$\mathcal{S}_{3} \Lambda^{3}$

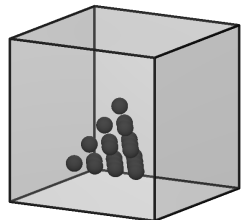

Legend: symbols represent the value or moment of the indicated quantities:

- - scalar (1 DOF); $\quad \boldsymbol{0}$ - tangential vector field (2 DOFs);

$\mathbf{\omega}$ - vector field (3 DOFs); $>$-tangential component of vector field on edge or normal component on face (1 DOF).

Figure 1. Element diagrams for $\mathcal{S}_{r} \Lambda^{k}$ in three dimensions for $r \leq 3$.

for each polynomial degree. Together with a corresponding discontinuous piecewise polynomial discretization of $L^{2}(\Omega)$, these RT spaces stably discretize the mixed variational formulation of the Poisson equation on $\Omega$. In [11, versions of the RT elements were given both for meshes of $\Omega$ by triangles and by rectangles. Besides the original application of mixed finite elements for the Poisson equation, the RT elements can be used together with the standard Lagrange finite element discretization of $H^{1}(\Omega)$ to give a stable mixed finite element discretization of the vector Poisson equation curl curl $u-\operatorname{grad} \operatorname{div} u=f$, in which the vector variable $u$ is sought in $H$ (div) and approximated by RT elements, and the scalar variable $\sigma=\operatorname{curl} u$ is sought in $H^{1}$ and approximated by Lagrange elements. The RT elements were generalized to three dimensions by Nédélec [9], with separate generalizations giving discretizations of $H(\operatorname{div}, \Omega)$ and of $H(\operatorname{curl}, \Omega)$, including both tetrahedral and cubic mesh generalizations of each. 
TABLE 1. Dimension of $\mathcal{S}_{r} \Lambda^{k}\left(I^{n}\right)$.

\begin{tabular}{|c|c|c|c|c|c|c|c|c|}
\hline & & & & & & & & \\
\hline & $k$ & 1 & 2 & 3 & 4 & 5 & 6 & 7 \\
\hline$n=1$ & 0 & 2 & 3 & 4 & 5 & 6 & 7 & 8 \\
\hline & 1 & 2 & 3 & 4 & 5 & 6 & 7 & 8 \\
\hline$n=2$ & 0 & 4 & 8 & 12 & 17 & 23 & 30 & 38 \\
\hline & 1 & 8 & 14 & 22 & 32 & 44 & 58 & 74 \\
\hline & 2 & 3 & 6 & 10 & 15 & 21 & 28 & 36 \\
\hline$n=3$ & 0 & 8 & 20 & 32 & 50 & 74 & 105 & 144 \\
\hline & 1 & 24 & 48 & 84 & 135 & 204 & 294 & 408 \\
\hline & 2 & 18 & 39 & 72 & 120 & 186 & 273 & 384 \\
\hline & 3 & 4 & 10 & 20 & 35 & 56 & 84 & 120 \\
\hline$n=4$ & 0 & 16 & 48 & 80 & 136 & 216 & 328 & 480 \\
\hline & 1 & 64 & 144 & 272 & 472 & 768 & 1188 & 1764 \\
\hline & 2 & 72 & 168 & 336 & 606 & 1014 & 1602 & 2418 \\
\hline & 3 & 32 & 84 & 180 & 340 & 588 & 952 & 1464 \\
\hline & 4 & 5 & 15 & 35 & 70 & 126 & 210 & 330 \\
\hline
\end{tabular}

In [7, Brezzi, Douglas, and Marini introduced a second family of finite element discretizations of $H(\operatorname{div}, \Omega)$ in two dimensions, for both triangular and rectangular meshes. These BDM elements have also proven to be very useful. Nédélec [10] generalized the BDM family to tetrahedral meshes in three dimensions, giving analogues both for $H$ (div) and $H$ (curl). He also defined $H($ div) and $H$ (curl) elements on cubic meshes in [10. However, these cannot really be considered analogues of the BDM elements, as they do not seem to lead to stable mixed finite element pairs. The generalization of the BDM elements to $H$ (div) on three-dimensional domains (but not $H($ curl) ), was also made by Brezzi, Douglas, Durán, and Fortin in [6] 1 The paper [6] also introduced an analogue of the rectangular BDM elements to cubic meshes in three dimensions.

The finite element exterior calculus [4, 5, has greatly clarified the relation of many of these mixed finite element methods. In exterior calculus the space $H$ (div) is viewed as a space of differential forms of degree $n-1$ in $n$ dimensions, while $H$ (curl) is a space of 1 -forms, $H^{1}$ a space of 0 -forms, and $L^{2}$ a space of $n$-forms. These spaces are connected via the de Rham complex, in which the fundamental differential operators grad, curl, div (and others in higher dimensions) are unified as the exterior derivative. We refer the reader to Table 2.2 in 4 for a summary

\footnotetext{
${ }^{1}$ The finite element discretization of $H($ div $)$ in [6] is identical with that of [10] in the case of tetrahedral meshes. However, the interior degrees of freedom differ. This does not affect the stability analysis for the mixed Poisson equation, but only the ones given by 10 can be used to establish stability when discretizing a mixed formulation of the vector Laplacian.
} 
of correspondences between differential forms and vector fields. In [4, 5] two fundamental families of finite element differential forms are defined on simplicial meshes in $n$ dimensions. Tables 5.1 and 5.2 in [4] summarize the correspondences between these spaces of finite element differential forms and classical finite element spaces in two and three dimensions. The $\mathcal{P}_{r}^{-} \Lambda^{k}$ family specializes to the Lagrange elements, the RT elements, and the fully discontinuous polynomial elements, for $k=0,1$, and 2, respectively, in two dimensions, and to the Lagrange elements, Nédélec's generalizations of the RT elements to $H$ (curl) and to $H$ (div), and the discontinuous elements for $k=0, \ldots, 3$ in three dimensions. Taken together, these spaces form a complex which is a finite element subcomplex of the de Rham complex:

$$
0 \rightarrow \mathcal{P}_{r}^{-} \Lambda^{0} \stackrel{d}{\rightarrow} \mathcal{P}_{r}^{-} \Lambda^{1} \stackrel{d}{\rightarrow} \cdots \stackrel{d}{\rightarrow} \mathcal{P}_{r}^{-} \Lambda^{n} \rightarrow 0 .
$$

The second family of finite elements discussed in [4,5] is the $\mathcal{P}_{r} \Lambda^{k}$ family. For 0forms and $n$-forms this brings nothing new, just recapturing the Lagrange and fully discontinuous polynomial elements, but for $0<k<n$, this is a different family of finite element spaces. For $n=2, k=1$ it gives the BDM triangular elements, and for $n=3, k=1$ and 2, it gives Nédélec's generalizations of these to $H$ (div) and $H$ (curl). They combine into a second finite element de Rham subcomplex

$$
0 \rightarrow \mathcal{P}_{r} \Lambda^{0} \stackrel{d}{\rightarrow} \mathcal{P}_{r-1} \Lambda^{1} \stackrel{d}{\rightarrow} \cdots \stackrel{d}{\rightarrow} \mathcal{P}_{r-n} \Lambda^{n} \rightarrow 0 .
$$

Note that the degree decreases in this complex, in contrast to the preceeding one.

We now turn to the construction of analogous spaces and complexes for cubical meshes. An analogue to the $\mathcal{P}_{r}^{-} \Lambda^{k}$ complex of elements for cubical meshes may be easily constructed via a tensor product construction. For an explicit description in $n$ dimensions and for all form degrees $k$, see [3]. This includes the tensor product Lagrange, or $\mathcal{Q}_{r}$, elements for 0 -forms, the rectangular RT elements for 1-forms in $2-\mathrm{D}$, and the 3-D generalizations of them given in [9]. The space for $n$-forms $\left(L^{2}\right)$ is the fully discontinuous space of tensor product polynomials (shape functions in $\left.\mathcal{Q}_{r}\right)$.

This paper develops a second family of finite element spaces for cubical meshes. This family may be viewed as an analogue of the $\mathcal{P}_{r} \Lambda^{k}$ family for cubical meshes. We denote the new family of elements by $\mathcal{S}_{r} \Lambda^{k}$ and define such a space for all dimensions $n \geq 1$, all polynomial degrees $r \geq 1$, and all form degrees $0 \leq k \leq n$. For 0 -forms (discretization of $H^{1}$ ), $S_{r} \Lambda^{0}$ is not equal to $\mathcal{Q}_{r}$, but rather to the serendipity elements in 2-D, their three-dimensional extension (which can be found in many places for small values of $r$ and for general $r$ in [12] and [8]), and to a recent extension to all dimensions [2]. For $n$-forms, $\mathcal{S}_{r} \Lambda^{n}$ uses fully discontinuous elements with shape functions in $\mathcal{P}_{r}\left(\right.$ not $\left.\mathcal{Q}_{r}\right)$.

For 1-forms in 2-D, the space $\mathcal{S}_{r} \Lambda^{1}$ coincides with the rectangular BDM elements of [7]. For 2-forms in 3-D, we believe that the space $\mathcal{S}_{r} \Lambda^{2}$, which has not appeared before as far as we know, is the correct analogue of the BDM elements on cubic meshes. It has the same degrees of freedom as the space given in [6] but the shape functions have better symmetry properties. For 1 -forms in $3-\mathrm{D}, \mathcal{S}_{r} \Lambda^{1}$ is a finite element discretization of $H$ (curl). To the best of our knowledge, neither the degrees of freedom nor the shape functions for this space have been proposed previously. Even for 0 -forms, the spaces $\mathcal{S}_{r} \Lambda^{0}$ were only discovered very recently in higher dimensions. They are the generalization of the serendipity spaces given by the present authors in 2]. That work was motivated by the search for a finite element 
discretization of the de Rham complex on cubical meshes which is completed in this paper.

In the remainder of the paper we will develop the $\mathcal{S}_{r} \Lambda^{k}$ spaces and their properties in the setting of differential forms on cubical meshes of arbitrary dimensions. Here we explicitly describe the spaces which arise in three dimensions in traditional finite element terminology by giving their shape functions and degrees of freedom. See also Figure 1

The space $\mathcal{S}_{r} \Lambda^{3}$. This is simply the space of piecewise polynomials of degree at most $r$ with no continuity requirements. Obviously this gives a finite element subspace of $L^{2}$ (which, unlike the remaining spaces, is defined also for $r=0$ ). Its dimension is $(r+1)(r+2)(r+3) / 6$.

The space $\mathcal{S}_{r} \Lambda^{2}$. The shape functions for the space $\mathcal{S}_{r} \Lambda^{2}$ on the unit cube $I^{3}$ in three dimensions are vector polynomials of the form

$$
u=\left(v_{1}, v_{2}, v_{3}\right)+\operatorname{curl}\left(x_{2} x_{3}\left(w_{2}-w_{3}\right), x_{3} x_{1}\left(w_{3}-w_{1}\right), x_{1} x_{2}\left(w_{1}-w_{2}\right)\right),
$$

with $v_{i}, w_{i} \in \mathcal{P}_{r}\left(I^{3}\right)$ and $w_{i}$ independent of $x_{i}$. The dimension of this space is $(r+1)\left(r^{2}+5 r+12\right) / 2$. As degrees of freedom for $u \in \mathcal{S}_{r} \Lambda^{2}$ we take

$$
u \mapsto \int_{f} u \cdot n p, p \in \mathcal{P}_{r}(f), f \text { a face of } I^{3} ; \quad u \mapsto \int_{I^{3}} u \cdot p, p \in\left[\mathcal{P}_{r-2}\left(I^{3}\right)\right]^{3} .
$$

The space $\mathcal{S}_{r} \Lambda^{1}$. The shape functions for the space $\mathcal{S}_{r} \Lambda^{1}$ in three dimensions are vector polynomials of the form

$$
u=\left(v_{1}, v_{2}, v_{3}\right)+\left(x_{2} x_{3}\left(w_{2}-w_{3}\right), x_{3} x_{1}\left(w_{3}-w_{1}\right), x_{1} x_{2}\left(w_{1}-w_{2}\right)\right)+\operatorname{grad} s,
$$

with $v_{i} \in \mathcal{P}_{r}\left(I^{3}\right), w_{i} \in \mathcal{P}_{r-1}\left(I^{3}\right)$ independent of $x_{i}$, and $s$ is a polynomial of $I^{3}$ with superlinear degree at most $r+1$, where the superlinear degree of a polynomial is the ordinary degree ignoring variables which enter linearly (e.g., the superlinear degree of $x_{1}^{2} x_{2} x_{3}^{3}$ is 5$)$. The dimension of this space is $(r+1)\left(r^{2}+5 r+18\right) / 2$. As degrees of freedom for $u \in \mathcal{S}_{r} \Lambda^{1}$ we take

$$
\begin{gathered}
u \mapsto \int_{e} u \cdot t p, p \in \mathcal{P}_{r}(e), e \text { an edge of } I^{3} ; \\
u \mapsto \int_{f} u \times n p, p \in\left[\mathcal{P}_{r-2}(f)\right]^{2}, f \text { a face of } I^{3} ; \quad u \mapsto \int_{I^{3}} u \cdot p, p \in\left[\mathcal{P}_{r-4}\left(I^{3}\right)\right]^{3} .
\end{gathered}
$$

The space $\mathcal{S}_{r} \Lambda^{0}$. Finally, the space $S_{r} \Lambda^{0}$ is the generalized serendipity space of 2. The shape functions are all polynomials of superlinear degree at most $r$, and the degrees of freedom are the values at the vertices and the moments of degree at most $r-2, r-4$, and $r-6$ on the edges, faces, and interior, respectively. The dimension of this space is 8 for $r=1,20$ for $r=2$, and $(r+1)\left(r^{2}+5 r+24\right) / 6$ for $r \geq 3$.

As a consequence of the general theory below, the degrees of freedom given are unisolvent for all these spaces, and for any cubical decomposition $\mathcal{T}$ of $\Omega$, the assembled finite element spaces $\mathcal{S}_{r} \Lambda^{k}(\mathcal{T})$ have exactly the continuity required to belong to $H^{1}(\Omega), H(\operatorname{div}, \Omega), H(\operatorname{curl}, \Omega)$, and $L^{2}(\Omega)$, for $k=0, \ldots, 3$, respectively. In other words, $\mathcal{S}_{r} \Lambda^{k}(\mathcal{T})$ belongs to the domain of the exterior derivative on $k$-forms. Moreover, exterior derivative maps $\mathcal{S}_{r} \Lambda^{k}(\mathcal{T})$ into $\mathcal{S}_{r-1} \Lambda^{k+1}(\mathcal{T})$, so we obtain a finite element subcomplex

$$
0 \rightarrow \mathcal{S}_{r} \Lambda^{0}(\mathcal{T}) \stackrel{\text { grad }}{\longrightarrow} \mathcal{S}_{r-1} \Lambda^{1}(\mathcal{T}) \stackrel{\text { curl }}{\longrightarrow} \mathcal{S}_{r-2} \Lambda^{2}(\mathcal{T}) \stackrel{\text { div }}{\longrightarrow} \mathcal{S}_{r-3} \Lambda^{3}(\mathcal{T}) \rightarrow 0
$$


of the de Rham complex. Finally, if we define projection operators $\pi^{k}$ from smooth fields into the finite element spaces $S_{r} \Lambda^{k}$ using the degrees of freedom, these commute with exterior differentiation. That is, the following diagram commutes:

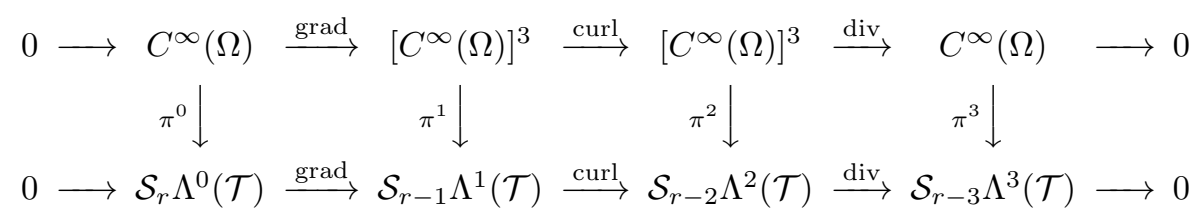

where, for simplicity, we assume $r \geq 3$ (otherwise some of the spaces are undefined and some parts of the diagram are not applicable).

The remainder of the paper consists of four sections. In Section 2, we recall some key concepts from exterior calculus, particularly the Koszul differential and Koszul complex of polynomial differential forms, which will be crucial to our construction. We introduce the concept of linear degree, and show that the subcomplex obtained from the Koszul complex by placing a lower bound on linear degree is exact. This is a key step in the unisolvence proof in Section 3. In Section 3, we define the spaces $\mathcal{S}_{r} \Lambda^{k}$ on an $n$-dimensional cube by giving the shape functions and degrees of freedom. We derive a number of properties from these definitions, leading to a formula for the dimension of the space of shape functions and a proof of unisolvence of the degrees of freedom. We then define projection operators $\pi_{r}^{k}$ mapping smooth fields into the finite element spaces $\mathcal{S}_{r} \Lambda^{k}$ and show that the projections commute with the exterior derivative. This accomplished, the new elements fit squarely into the framework of the finite element exterior calculus given in [5]. Therefore the application of the elements to PDE problems and their numerical analysis does not require new ideas, and so we do not discuss that here. The proof of unisolvence in Section 3 hinges on the special case of functions with vanishing trace. The proof in that case is the topic of Section 4.

\section{NotATION AND PRELIMINARIES}

For $n \geq 1$, the number of dimensions, let $\mathbb{N}_{n}=\{1, \ldots, n\}$, and let $\Sigma(k), 0 \leq$ $k \leq n$, denote the set of subsets of $\mathbb{N}_{n}$ consisting of $k$ elements. For $\sigma \in \Sigma(k)$ we denote by $\sigma^{*} \in \Sigma(n-k)$ its complement $\mathbb{N}_{n} \backslash \sigma$. For $p \in \sigma$ we write $\sigma-p$ for $\sigma \backslash\{p\} \in \Sigma(k-1)$, and for $q \in \sigma^{*}$ we write $\sigma+q$ for $\sigma \cup\{q\} \in \Sigma(k+1)$. If $\sigma \subset \mathbb{N}_{n}$ and $q \in \sigma^{*}$ we let $\epsilon(q, \sigma)=(-1)^{l}$ where $l=\#\{p \in \sigma \mid p<q\}$, and set $\epsilon(q, p, \sigma):=\epsilon(q, \sigma) \epsilon(p, \sigma+q-p)$ for $q \in \sigma^{*}, p \in \sigma$. For later reference, we note that

$$
\epsilon(q, p, \sigma)=-\epsilon(p, \sigma-p) \epsilon(q, \sigma-p),
$$

which is easily verified by considering the cases $q<p$ and $q>p$ separately.

We now recall some basic tools and results of exterior algebra and exterior calculus. These can be found, for example, in [4, Section 2]. For each $\sigma \in \Sigma(k)$ we denote by $\sigma_{1}, \ldots, \sigma_{k}$ its elements in increasing order, and by

$$
d x_{\sigma}=d x_{\sigma_{1}} \wedge \cdots \wedge d x_{\sigma_{k}}
$$

the corresponding basic alternator. A differential $k$-form $\omega$ on a domain $\Omega \subset \mathbb{R}^{n}$ may be written as

$$
\omega=\sum_{\sigma \in \Sigma(k)} \omega_{\sigma} d x_{\sigma}
$$


with coefficients $\omega_{\sigma}$ belonging to any desired space of functions on $\Omega$, e.g., $L^{2}(\Omega)$. A 0 -form is simply such a function. The exterior derivative of the differential form (41) is

$$
d \omega=\sum_{\sigma \in \Sigma(k)} \sum_{q \in \mathbb{N}_{n}} \partial_{q} \omega_{\sigma} d x_{q} \wedge d x_{\sigma}
$$

where $\partial_{q}=\partial / \partial x_{q}$.

A differential $k$-form may be contracted with a vector field on $\Omega$ to give a differential $(k-1)$-form (or zero if $k=0$ ). When the vector field is simply the identity, the resulting operator is the Koszul differential. Equivalently, we may define the Koszul differential on the basic alternators by

$$
\kappa\left(d x_{\sigma_{1}} \wedge \cdots \wedge d x_{\sigma_{k}}\right)=\sum_{i=1}^{k}(-1)^{i+1} x_{\sigma_{i}} d x_{\sigma_{1}} \wedge \cdots \wedge \widehat{d x_{\sigma_{i}}} \wedge \cdots \wedge d x_{\sigma_{k}},
$$

and then extend it to a general differential form by linearity:

$$
\kappa\left(\sum_{\sigma} \omega_{\sigma} d x_{\sigma}\right)=\sum_{\sigma} \omega_{\sigma} \sum_{i=1}^{k}(-1)^{i+1} x_{\sigma_{i}} d x_{\sigma_{1}} \wedge \cdots \wedge \widehat{d x_{\sigma_{i}}} \wedge \cdots \wedge d x_{\sigma_{k}} .
$$

The operator $\kappa$ is a graded differential, meaning that

$$
\kappa(\kappa \omega)=0, \quad \kappa(\omega \wedge \eta)=\kappa \omega \wedge \eta+(-1)^{k} \omega \wedge \kappa \eta,
$$

if $\omega$ is a $k$-form and $\eta$ an $l$-form.

The following lemma collects formulas for $\kappa \omega, d \omega$, and $\kappa d \omega$.

Lemma 2.1. If $\omega$ is given by (4), then

$$
\begin{aligned}
\kappa \omega & =\sum_{\zeta \in \Sigma(k-1)} \eta_{\zeta} d x_{\zeta}, \text { where } \eta_{\zeta}=\sum_{q \in \zeta^{*}} \epsilon(q, \zeta) x_{q} \omega_{\zeta+q}, \\
\text { (7) } \quad d \omega & =\sum_{\rho \in \Sigma(k+1)} \nu_{\rho} d x_{\rho}, \text { where } \nu_{\rho}=\sum_{q \in \rho} \epsilon(q, \rho-q) \partial_{q} \omega_{\rho-q}, \\
\text { (8) } \quad \kappa d \omega= & \sum_{\sigma \in \Sigma(k)} \mu_{\sigma} d x_{\sigma}, \text { where } \mu_{\sigma}=\sum_{q \in \sigma^{*}}\left[x_{q} \partial_{q} \omega_{\sigma}+\sum_{p \in \sigma} \epsilon(q, p, \sigma) x_{q} \partial_{p} \omega_{\sigma+q-p}\right] .
\end{aligned}
$$

Proof. By definition

$$
\kappa d x_{\sigma}=\sum_{q \in \sigma} \epsilon(q, \sigma-q) x_{q} d x_{\sigma-q}
$$

SO

$$
\kappa \omega=\sum_{\sigma \in \Sigma(k)} \sum_{q \in \sigma} \epsilon(q, \sigma-q) x_{q} \omega_{\sigma} d x_{\sigma-q} .
$$

Making the change of variable $\zeta=\sigma-q$, so

$$
\sigma \in \Sigma(k), \quad q \in \sigma \quad \Longleftrightarrow \quad \zeta \in \Sigma(k-1), \quad q \in \zeta^{*},
$$

we obtain (6). The second result is proven similarly, and the third follows from the first two.

We now turn to differential forms with polynomial coefficients. A monomial in $n$ variables is determined by a multi-index $\alpha$ of $n$ nonnegative integers: $x^{\alpha}=$ $x_{1}^{\alpha_{1}} \cdots x_{n}^{\alpha_{n}}$. By a form monomial in $n$ variables, we mean the product of a monomial 
with a basic alternator: $m=x^{\alpha} d x_{\sigma}$ for some multi-index $\alpha$ and $\sigma \subset \mathbb{N}_{n}$. The polynomial degree and the linear degree of $m$ are defined as

$$
\operatorname{deg} m=|\alpha|:=\sum_{i} \alpha_{i}, \quad \operatorname{ldeg} m=\#\left\{i \in \sigma^{*} \mid \alpha_{i}=1\right\} .
$$

Thus the linear degree of $m$ is the degree of its polynomial coefficient counting only those variables which enter linearly, and excluding variables which enter the alternator. For ordinary monomials, i.e., 0-forms, the linear degree is equal to the difference between the polynomial degree and the superlinear degree which appeared in the introduction.

We define $\mathcal{H}_{r} \Lambda^{k}=\mathcal{H}_{r} \Lambda^{k}\left(\mathbb{R}^{n}\right)$ to be the span of the $k$-form monomials $m$ with $\operatorname{deg} m=r$, and $\mathcal{P}_{r} \Lambda^{k}=\mathcal{P}_{r} \Lambda^{k}\left(\mathbb{R}^{n}\right)=\sum_{s=0}^{r} \mathcal{H}_{s} \Lambda^{k}$ to be the span of those with $\operatorname{deg} m \leq r$. If $k=0$, we may simply write $\mathcal{H}_{r}$ and $\mathcal{P}_{r}$. If $\Omega$ is a subdomain of $\mathbb{R}^{n}$, we define $\mathcal{P}_{r} \Lambda^{k}(\Omega)$ to be the space of restrictions to $\Omega$ of the elements of $\mathcal{P}_{r} \Lambda^{k}$ (and similarly for other function spaces). Note that $d$ maps $\mathcal{H}_{r} \Lambda^{k}$ into $\mathcal{H}_{r-1} \Lambda^{k+1}$ while $\kappa$ maps $\mathcal{H}_{r} \Lambda^{k}$ into $\mathcal{H}_{r+1} \Lambda^{k-1}$. An extremely useful identity is the homotopy formula ([4, Theorem 3.1]):

$$
(d \kappa+\kappa d) \omega=(r+k) \omega, \quad \omega \in \mathcal{H}_{r} \Lambda^{k}\left(\mathbb{R}^{n}\right) .
$$

We extend the linear degree for form monomials to polynomial differential forms by defining $\operatorname{ldeg} \mu$ for any $\mu \in \mathcal{H}_{r} \Lambda^{k}$ to be the minimum of the linear degree among all the monomials $m$ in $\mu$. We say that $\mu$ is of homogeneous linear degree equal to $l$ if $\operatorname{ldeg} m=l$ for every monomial of $\mu$. We denote by $\mathcal{H}_{r, l} \Lambda^{k}$ the space of forms in $\mathcal{H}_{r} \Lambda^{k}$ of linear degree at least $l$. Obviously,

$$
\mathcal{H}_{r, l} \Lambda^{k}\left(\mathbb{R}^{n}\right)=0, \quad l>\min (r, n-k) .
$$

The exterior derivative $d$ may decrease the linear degree of a polynomial differential form, but $\kappa$ and $\kappa d \kappa$ do not.

Lemma 2.2. For any $\omega \in \mathcal{P}_{r} \Lambda^{k}$, $\operatorname{ldeg} \kappa \omega \geq \operatorname{ldeg} \omega$ and $\operatorname{ldeg} \kappa d \kappa \omega \geq \operatorname{ldeg} \omega$.

Proof. If $m$ is monomial of $\omega$ and $l=\operatorname{ldeg} m$, then it follows directly from the definition (5) that the monomials of $\kappa m$ are of linear degree $l$ and/or $l+1$, so $\operatorname{ldeg} \kappa m \geq l \geq \operatorname{ldeg} \omega$. Since every monomial of $\kappa \omega$ is a monomial of $\kappa m$ for some monomial $m$ of $\omega$, this implies the first inequality. For the second we use the differential property of $\kappa$ and the homotopy formula to see that $\kappa d \kappa m=(\kappa d+$ $d \kappa) \kappa m$ is a multiple of $\kappa m$. Therefore $\operatorname{ldeg} \kappa d \kappa m=\operatorname{ldeg} \kappa m \geq \operatorname{ldeg} \omega$, which gives the second inequality.

In view of Lemma 2.2 for each $l \geq 0$, we obtain a complex

$$
\cdots \stackrel{\kappa}{\rightarrow} \mathcal{H}_{r-1, l} \Lambda^{k+1} \stackrel{\kappa}{\rightarrow} \mathcal{H}_{r, l} \Lambda^{k} \stackrel{\kappa}{\rightarrow} \cdots .
$$

When $l=0$ this is the Koszul complex, and exactness follows from the homotopy formula. In fact, the complex (11) is exact for all $l \geq 0$.

Theorem 2.3. For $r \geq 1,0 \leq l<r, 0 \leq k<n$, the sequence

$$
\mathcal{H}_{r-1, l} \Lambda^{k+1} \stackrel{\kappa}{\rightarrow} \mathcal{H}_{r, l} \Lambda^{k} \stackrel{\kappa}{\rightarrow} \mathcal{H}_{r+1, l} \Lambda^{k-1}
$$

is exact. Equivalently, $\operatorname{dim} \kappa\left(\mathcal{H}_{r-1, l} \Lambda^{k+1}\right)+\operatorname{dim} \kappa\left(\mathcal{H}_{r, l} \Lambda^{k}\right)=\operatorname{dim} \mathcal{H}_{r, l} \Lambda^{k}$.

The proof is due to Scot Adams and Victor Reiner [1. Its main ingredient is contained in the following lemma. 
Lemma 2.4. Let $r \geq 1, l \geq 1$, and $0 \leq k<n$. Suppose that $\mu \in \mathcal{H}_{r} \Lambda^{k}$ is of linear degree at least $l-1$ and $\kappa \mu$ is of linear degree at least $l$. Then there exists $\nu \in \mathcal{H}_{r-1} \Lambda^{k+1}$ such that $\mu-\kappa \nu$ is of linear degree at least l. Further, if $\mu \in \mathcal{H}_{r} \Lambda^{n}$ is nonzero, then $\kappa \mu$ is of linear degree 0 .

Proof. For the final statement, concerning $n$-forms, we write $\mu=p d x_{1} \wedge \cdots \wedge d x_{n}$ where $p \in \mathcal{H}_{r}$. Since $r \geq 1, p$ is not constant. But then $\kappa \mu=\sum_{i}(-1)^{i+1} p x_{i} d x_{1} \wedge$ $\cdots \wedge \widehat{d x}_{i} \wedge \cdots \wedge d x_{n}$ is easily seen to be of linear degree 0 .

For $0 \leq k<n$, the proof hinges on a canonical form for an element of $\mu \in \mathcal{H}_{r} \Lambda^{k}$ which we establish before proceeding. Let us say that a form monomial $x^{\alpha} d x_{\sigma}$ is full if $\sigma \subset \operatorname{supp}(\alpha)$, the support of $\alpha$. To each of the monomials $m=x^{\alpha} d x_{\sigma}$ of $\mu \in \mathcal{H}_{r} \Lambda^{k}$, we associate the increasing sequences $\rho$ and $\tau$ with $\rho=\sigma \cap \operatorname{supp}(\alpha)$ and $\tau=\sigma \backslash \operatorname{supp}(\alpha)$. Then $m= \pm \eta \wedge d x_{\tau}$ where $\eta=x^{\alpha} d x_{\rho}$ is a full form monomial which is independent of the $\tau$ variables (that is, $\operatorname{supp}(\alpha) \cup \rho$ is disjoint from $\tau$ ). Note that $\operatorname{ldeg} \eta=\operatorname{ldeg} m$. Finally, in the expansion of $\mu$ as a linear combination of its monomials, we gather together the terms with the same $\tau=\sigma \backslash \operatorname{supp}(\alpha)$, and in this way write

$$
\mu=\sum_{\tau \subset \mathbb{N}_{n}} \eta_{\tau} \wedge d x_{\tau}
$$

where $\eta_{\tau} \in \mathcal{H}_{r} \Lambda^{k-\# \tau}$ is independent of the $\tau$ variables, and has all of its monomials full. The expression on the right-hand side of (12) is the desired canonical form of $\mu$.

Now we proceed with the proof of the lemma. We consider first the special case in which $\mu$ is of homogeneous linear degree $l-1$, and, in this special case, we use induction on $l$, the case $l=0$ being known (exactness of the Koszul complex). Expressing $\mu$ in the canonical form (12), we have $\operatorname{ldeg} \eta_{\tau}=l-1$ for each $\tau$ (for which the coefficient $\eta_{\tau}$ does not vanish). Now

$$
\kappa \mu=\sum \kappa \eta_{\tau} \wedge d x_{\tau}+\sum \pm \eta_{\tau} \wedge \kappa d x_{\tau} .
$$

The first sum is of homogeneous linear degree $l-1$ and the second of homogeneous linear degree $l$. Since we assumed that $\kappa \mu$ is of linear degree at least $l$, the first sum must vanish. But this sum is in canonical form, so we conclude that $\kappa \eta_{\tau}=0$ for each $\tau$. Invoking the inductive hypothesis, we can write $\eta_{\tau}=\kappa \nu_{\tau}$ where $\nu_{\tau}$ has linear degree at least $l-1$. Let

$$
\nu=\sum \nu_{\tau} \wedge d x_{\tau} \in \mathcal{H}_{r-1} \Lambda^{k+1} .
$$

Then

$$
\mu-\kappa \nu=\sum \eta_{\tau} \wedge d x_{\tau}-\sum \kappa \nu_{\tau} \wedge d x_{\tau}-\sum \pm \nu_{\tau} \wedge \kappa d x_{\tau}=-\sum \pm \nu_{\tau} \wedge \kappa d x_{\tau}
$$

Expanding the right-hand side into monomials, we see that each has ldeg at least $l$, so this completes the proof under the assumption that $\mu$ is of homogeneous linear degree $l-1$.

Next we turn to the general case, in which $\mu$ is of linear degree at least $l-1$. We may split $\mu$ as $\mu^{\prime}+\mu^{\prime \prime}$ with $\mu^{\prime}$ of homogeneous linear degree $l-1$ and $\mu^{\prime \prime}$ of linear degree at least $l$. Then $\kappa \mu^{\prime}$ splits into a part of homogeneous linear degree $l-1$ and a part of homogeneous linear degree $l$, while $\operatorname{ldeg}\left(\kappa \mu^{\prime \prime}\right) \geq l$. Since, by assumption, $\operatorname{ldeg}(\kappa \mu) \geq l$, the part of $\kappa \mu^{\prime}$ with linear degree equal to $l-1$ must vanish. That is, $\operatorname{ldeg}\left(\kappa \mu^{\prime}\right) \geq l$. Therefore, we may apply the result of the preceding special case to $\mu^{\prime}$ 
to obtain $\nu \in \mathcal{H}_{r-1} \Lambda^{k+1}$ such that $\operatorname{ldeg}\left(\mu^{\prime}-\kappa \nu\right) \geq l$. Then $\mu-\kappa \nu=\left(\mu^{\prime}-\kappa \nu\right)+\mu^{\prime \prime}$ is of linear degree at least $l$.

Finally, we give the proof of Theorem 2.3 .

Proof. The result is certainly true for $l=0$, so we may assume $1 \leq l<r$ (and so $r \geq 2$ ). Suppose $\omega \in \mathcal{H}_{r, l} \Lambda^{k}$ with $\kappa \omega=0$. We must show that there exists $\eta \in$ $\mathcal{H}_{r-1} \Lambda^{k+1}$ with linear degree at least $l$, such that $\kappa \eta=\omega$. Now $\mu:=d \omega /(r+k) \in$ $\mathcal{H}_{r-1} \Lambda^{k+1}$ is of linear degree at least $l-1$ and satisfies $\kappa \mu=(\kappa d+d \kappa) \omega /(r+k)=\omega$ by (9). If $k=n-1$, the final sentence of the lemma insures that $\mu=0$. For $0 \leq k<n-1$, we apply the lemma with $r$ and $k$ replaced by $r-1$ and $k+1$, respectively, and conclude that there exists $\nu \in \mathcal{H}_{r-2} \Lambda^{k+2}$ such that $\eta:=\mu-\kappa \nu$ is of linear degree at least $l$. Clearly, $\kappa \eta=\kappa \mu=\omega$.

\section{THE $\mathcal{S}_{r} \Lambda^{k}$ SPACES}

Here, the main section of the paper, we define the polynomial spaces $\mathcal{S}_{r} \Lambda^{k}\left(\mathbb{R}^{n}\right)$ we shall use as shape functions (see (17) below) and the degrees of freedom for these (see (21)). We derive a number of properties of these polynomial spaces in Theorems 3.2 through 3.5 and use them to verify unisolvence in Theorem 3.6.

The space of shape functions will consist of polynomials of a given degree plus certain additional terms of higher degree which will be defined in terms of the following auxilliary space:

$$
\mathcal{J}_{r} \Lambda^{k}\left(\mathbb{R}^{n}\right)=\sum_{l \geq 1} \kappa \mathcal{H}_{r+l-1, l} \Lambda^{k+1}\left(\mathbb{R}^{n}\right) .
$$

In view of (10), the sum is finite and

$$
\mathcal{J}_{r} \Lambda^{k}\left(\mathbb{R}^{n}\right) \subset \mathcal{P}_{r+n-k-1} \Lambda^{k}\left(\mathbb{R}^{n}\right) .
$$

Moreover, the sum is direct, since the polynomial degrees of the summands differ. The following proposition, which follows directly from the definitions, helps to clarify the meaning of this space.

Proposition 3.1. 1. The space $\sum_{l \geq 1} \mathcal{H}_{r+l-1, l} \Lambda^{k}\left(\mathbb{R}^{n}\right)$ is the span of all $k$-form monomials $m$ with $\operatorname{deg} m \geq r$ and $\operatorname{deg} m-\operatorname{ldeg} m \leq r-1$.

2. The space $\mathcal{J}_{r} \Lambda^{k}\left(\mathbb{R}^{n}\right)$ is the span of $\kappa m$ for all $(k+1)$-form monomials $m$ with $\operatorname{deg} m \geq r$ and $\operatorname{deg} m-\operatorname{ldeg} m \leq r-1$.

For several values of $k$, this space can be described more explicitly. By (10),

$$
\mathcal{J}_{r} \Lambda^{k}\left(\mathbb{R}^{n}\right)=0, \text { for } k=n \text { or } n-1,
$$

while

$$
\mathcal{J}_{r} \Lambda^{n-2}\left(\mathbb{R}^{n}\right)=\kappa \mathcal{H}_{r, 1} \Lambda^{n-1}\left(\mathbb{R}^{n}\right) .
$$

Now, $\mathcal{H}_{r, 1} \Lambda^{n-1}\left(\mathbb{R}^{n}\right)$ is the span of the monomials $x_{i} w_{i} \theta_{i}$, where $w_{i} \in \mathcal{H}_{r-1}\left(\mathbb{R}^{n}\right)$ is independent of $x_{i}$ and $\theta_{i}:=(-1)^{i-1} d x_{1} \wedge \ldots \wedge \widehat{d x^{i}} \wedge \ldots \wedge d x_{n}$. We then have

$$
\kappa \theta_{i}=\sum_{j<i} x_{j} \theta_{j, i}-\sum_{j>i} x_{j} \theta_{i, j}
$$

with

$$
\theta_{i, j}=(-1)^{i+j} d x_{1} \wedge \ldots \wedge \widehat{d x^{i}} \wedge \ldots \wedge \widehat{d x^{j}} \wedge \ldots \wedge d x_{n}
$$


Therefore,

$$
\mathcal{J}_{r} \Lambda^{n-2}\left(\mathbb{R}^{n}\right)=\left\{\sum_{i<j} x_{i} x_{j}\left(w_{i}-w_{j}\right) \theta_{i, j} \mid w_{i} \in \mathcal{H}_{r-1}\left(\mathbb{R}^{n}\right) \text { independent of } x_{i}\right\}
$$

Finally, we identify $\mathcal{J}_{r} \Lambda^{0}\left(\mathbb{R}^{n}\right)$. By Theorem 2.3 in the case $k=0$, we see that $\mathcal{J}_{r} \Lambda^{0}\left(\mathbb{R}^{n}\right)=\sum_{l \geq 1} \mathcal{H}_{r+l, l} \Lambda^{0}\left(\mathbb{R}^{n}\right)$. By Proposition 3.1. this space is the span of monomials of degree $>r$ whose superlinear degree, that is, deg - ldeg, is at most $r$.

We can now define the space of polynomial $k$-forms which we use for shape functions,

$$
\mathcal{S}_{r} \Lambda^{k}\left(\mathbb{R}^{n}\right):=\mathcal{P}_{r} \Lambda^{k}\left(\mathbb{R}^{n}\right)+\mathcal{J}_{r} \Lambda^{k}\left(\mathbb{R}^{n}\right)+d \mathcal{J}_{r+1} \Lambda^{k-1}\left(\mathbb{R}^{n}\right),
$$

defined for all $r \geq 1$ and all $0 \leq k \leq n$. From (14),

$$
\mathcal{S}_{r} \Lambda^{k}\left(\mathbb{R}^{n}\right) \subset \mathcal{P}_{r+n-k} \Lambda^{k}\left(\mathbb{R}^{n}\right) .
$$

Note that, in case $k=0$, the final term in (17) vanishes, and, by the characterization of $\mathcal{J}_{r} \Lambda^{0}\left(\mathbb{R}^{n}\right)$ just derived, $\mathcal{S}_{r} \Lambda^{0}\left(\mathbb{R}^{n}\right)$ consists precisely of the span of all monomials of superlinear degree at most $r$. This is exactly the serendipity space as defined in [2]. In this case, (14) gives the sharper degree bound

$$
\mathcal{S}_{r} \Lambda^{0}\left(\mathbb{R}^{n}\right) \subset \mathcal{P}_{r+n-1} \Lambda^{0}\left(\mathbb{R}^{n}\right) .
$$

Another case in which the expression for $\mathcal{S}_{r} \Lambda^{k}\left(\mathbb{R}^{n}\right)$ can be simplified is when $k=n$. By (15),

$$
\mathcal{S}_{r} \Lambda^{n}\left(\mathbb{R}^{n}\right)=\mathcal{P}_{r} \Lambda^{n}\left(\mathbb{R}^{n}\right) .
$$

When $k=n-1$, we have, by (15),

$$
\mathcal{S}_{r} \Lambda^{n-1}\left(\mathbb{R}^{n}\right):=\mathcal{P}_{r} \Lambda^{n-1}\left(\mathbb{R}^{n}\right)+d \mathcal{J}_{r+1} \Lambda^{n-2}\left(\mathbb{R}^{n}\right)
$$

where the last space is characterized in (16). In the case of three dimensions, this is formula (11) given in the introduction, stated in the language of exterior calculus. In a similar way, we recover formula (2) for the 3-D $H$ (curl) elements discussed in the introduction.

We now derive several properties of these polynomial spaces. The first limits the monomials that appear in the polynomials in $\mathcal{S}_{r} \Lambda^{k}\left(\mathbb{R}^{n}\right)$.

Theorem 3.2 (Degree property). For any $n, r \geq 1$ and $0 \leq k \leq n$, the space $\mathcal{S}_{r} \Lambda^{k}\left(\mathbb{R}^{n}\right)$ is contained in the span of the $k$-form monomials $m$ of degree at most $r+n-k-\delta_{k 0}$ for which

$$
\operatorname{deg} m-\operatorname{ldeg} m \leq r+1-\delta_{k 0} .
$$

Proof. The bound $r+n-k-\delta_{k 0}$ on the degree is given in (18) for $k>0$ and in (19) for $k=0$, so we need only show (20). If $m$ is a monomial of an element of $\mathcal{P}_{r} \Lambda^{k}\left(\mathbb{R}^{n}\right)$, then $\operatorname{deg} m \leq r$ and $\operatorname{ldeg} m \geq 0$, so (20) holds with $r$ on the righthand side. If $m$ is a monomial of an element of $\mathcal{J}_{r} \Lambda^{k}\left(\mathbb{R}^{n}\right)$, then $m$ occurs in the expansion of $\kappa p$, where $p$ is a $(k+1)$-form monomial with $\operatorname{deg} p-\operatorname{ldeg} p \leq r-1$ (Proposition 3.1). Then $\operatorname{deg} m=\operatorname{deg} p+1$ and, by Lemma 2.2, $\operatorname{ldeg} m \geq \operatorname{ldeg} p$, so again $\operatorname{deg} m-\operatorname{ldeg} m \leq r$. Finally, if $k>0$ and $m$ is a monomial of an element of $d \mathcal{J}_{r+1} \Lambda^{k-1}\left(\mathbb{R}^{n}\right)$, then by the argument just given, $m$ is a monomial of $d q$ where $q$ is a $(k-1)$-form monomial with $\operatorname{deg} q-\operatorname{ldeg} q \leq r+1$. Since $\operatorname{deg} m=\operatorname{deg} q-1$ and $\operatorname{ldeg} m \geq \operatorname{ldeg} q-1$, we get (20). 
A crucial property of these polynomial form spaces, is that they can be combined to form a subcomplex of the de Rham complex.

Theorem 3.3 (Subcomplex property). Let $n, r \geq 1$, and let $0<k \leq n$. Then

$$
d \mathcal{S}_{r+1} \Lambda^{k-1}\left(\mathbb{R}^{n}\right) \subset \mathcal{S}_{r} \Lambda^{k}\left(\mathbb{R}^{n}\right) .
$$

Proof. With reference to (17), we note that $d \mathcal{P}_{r+1} \Lambda^{k-1}\left(\mathbb{R}^{n}\right) \subset \mathcal{P}_{r} \Lambda^{k}\left(\mathbb{R}^{n}\right)$, and $d\left(d \mathcal{J}_{r+2} \Lambda^{k}\left(\mathbb{R}^{n}\right)\right)$ vanishes, so it suffices to prove that $d \mathcal{J}_{r+1} \Lambda^{k-1}\left(\mathbb{R}^{n}\right) \subset \mathcal{S}_{r} \Lambda^{k}\left(\mathbb{R}^{n}\right)$, which is immediate from (17).

We next observe that the spaces increase with increasing polynomial degree.

Theorem 3.4 (Inclusion property). Let $n, r \geq 1$, and let $0 \leq k \leq n$. Then

$$
\mathcal{S}_{r} \Lambda^{k}\left(\mathbb{R}^{n}\right) \subset \mathcal{S}_{r+1} \Lambda^{k}\left(\mathbb{R}^{n}\right) .
$$

Proof. We must show that each of the three summands on the right-hand side of (17) is included in $\mathcal{S}_{r+1} \Lambda^{k}\left(\mathbb{R}^{n}\right)$. Clearly,

$$
\mathcal{P}_{r} \Lambda^{k}\left(\mathbb{R}^{n}\right) \subset \mathcal{P}_{r+1} \Lambda^{k}\left(\mathbb{R}^{n}\right) \subset \mathcal{S}_{r+1} \Lambda^{k}\left(\mathbb{R}^{n}\right),
$$

which establishes the first inclusion. Next, we show that

$$
\mathcal{J}_{r} \Lambda^{k}\left(\mathbb{R}^{n}\right) \subset \mathcal{P}_{r+1} \Lambda^{k}\left(\mathbb{R}^{n}\right)+\mathcal{J}_{r+1} \Lambda^{k}\left(\mathbb{R}^{n}\right) \subset \mathcal{S}_{r+1} \Lambda^{k}\left(\mathbb{R}^{n}\right) .
$$

By Proposition 3.1 elements of $\mathcal{J}_{r} \Lambda^{k}\left(\mathbb{R}^{n}\right)$ are of form $\kappa m$ with $m$ a $(k+1)$-form monomial with $\operatorname{deg} m \geq r, \operatorname{deg} m-\operatorname{ldeg} m \leq r-1$. By the homotopy formula (9), $\kappa m$ is a constant multiple of $\kappa p$ with $p=d \kappa m$. We have $\operatorname{deg} p=\operatorname{deg} m$ and $\operatorname{ldeg} p \geq$ $\operatorname{ldeg} m-1$. Then $\operatorname{deg} p-\operatorname{ldeg} p \leq \operatorname{deg} m-\operatorname{ldeg} m+1 \leq r$. If $\operatorname{deg} p=r, \operatorname{deg} \kappa m=$ $r+1$ and $\kappa m \in \mathcal{P}_{r+1} \Lambda^{k}\left(\mathbb{R}^{n}\right)$. On the other hand, if $\operatorname{deg} p \geq r+1$, by Proposition [3.1, $\kappa m \in \mathcal{J}_{r+1} \Lambda^{k}\left(\mathbb{R}^{n}\right)$. This establishes the second inclusion. To complete the proof, we show that $d \mathcal{J}_{r+1} \Lambda^{k-1}\left(\mathbb{R}^{n}\right) \subset \mathcal{S}_{r+1} \Lambda^{k}\left(\mathbb{R}^{n}\right)$. Since $\mathcal{J}_{r+1} \Lambda^{k-1}\left(\mathbb{R}^{n}\right) \subset$ $\mathcal{S}_{r+2} \Lambda^{k-1}\left(\mathbb{R}^{n}\right)$ (by the inclusion just established), we infer from the subcomplex property that $d \mathcal{J}_{r+1} \Lambda^{k-1}\left(\mathbb{R}^{n}\right) \subset d \mathcal{S}_{r+2} \Lambda^{k-1}\left(\mathbb{R}^{n}\right) \subset \mathcal{S}_{r+1} \Lambda^{k}\left(\mathbb{R}^{n}\right)$.

The third property of the $\mathcal{S}_{r} \Lambda^{k}$ spaces that we establish concerns traces on hyperplanes. Consider a hyperplane $f$ of $\mathbb{R}^{n}$ of the form $x_{i}=c$ for some $1 \leq i \leq n$ and some constant $c$. The variables $x_{j}, j \neq i$, form a coordinate system for $f$, so we may identify $f$ with $\mathbb{R}^{n-1}$ and consider the space $\mathcal{S}_{r} \Lambda^{k}(f)$. It is a space of polynomial $k$-forms on $f$, and so vanishes if $k=n$. Next, we consider the trace on $f$ of a differential form in $n$ variables (defined as the pullback of the form through the inclusion map $\left.f \hookrightarrow \mathbb{R}^{n}\right)$. Let $\sigma \in \Sigma(k)$, and let $\omega_{\sigma}$ be a function of $n$ variables. Then

$$
\operatorname{tr}_{f}\left(\omega_{\sigma} d x_{\sigma}\right)= \begin{cases}0, & i \in \sigma, \\ \left(\operatorname{tr}_{f} \omega_{\sigma}\right) d x_{\sigma}, & i \notin \sigma .\end{cases}
$$

In the last expression, $\operatorname{tr}_{f} \omega_{\sigma}$ denotes the function of $n-1$ variables obtained by setting $x_{i}=c$ and we view $d x_{\sigma}$ as a basic alternator in the $n-1$ variables $x_{j}, j \neq i$. The trace property states that if $u \in \mathcal{S}_{r} \Lambda^{k}\left(\mathbb{R}^{n}\right)$, then $\operatorname{tr}_{f} u$, which is a polynomial $k$-form on $f$, belongs to $\mathcal{S}_{r} \Lambda^{k}(f)$.

Theorem 3.5 (Trace property). Let $n, r \geq 1,0 \leq k \leq n$, and let $f$ be a hyperplane of $\mathbb{R}^{n}$ obtained by fixing one coordinate. Then

$$
\operatorname{tr}_{f} \mathcal{S}_{r} \Lambda^{k}\left(\mathbb{R}^{n}\right) \subset \mathcal{S}_{r} \Lambda^{k}(f) .
$$

(This inclusion will be shown to be an equality in (27) below.) 
Proof. Without loss of generality, we assume that $f=\left\{x \in \mathbb{R}^{n} \mid x_{1}=c\right\}$. First let us comment on the Koszul operator applied to a polynomial differential form on $f$. Such a form may be written as a linear combination of monomials $x^{\alpha} d x_{\sigma}$ where $\alpha_{1}=0$ and $1 \notin \sigma$. Referring to (5) we see that, if we view $x^{\alpha} d x_{\sigma}$ as a form monomial in $n$ variables and take the Koszul differential, the result is the same as if we view it as form monomial in $n-1$ variables and take the Koszul differential. Thus we need not distinguish between the Koszul differential on $\mathbb{R}^{n}$ and that on $f$.

We will prove the theorem by induction on $k$. For $k=0$ we recall that $\mathcal{S}_{r} \Lambda^{0}\left(\mathbb{R}^{n}\right)$ is the serendipity space spanned by the monomials of superlinear degree at most $r$, and, of course, the superlinear degree does not increase when taking the trace. Hence, $\operatorname{tr}_{f} \mathcal{S}_{r} \Lambda^{0}\left(\mathbb{R}^{n}\right) \subset \mathcal{S}_{r} \Lambda^{0}(f)$.

To prove the theorem for $k>0$, assume that it holds with $k$ replaced by $k-1$. In light of (17) we need to show that the traces of each of the three spaces $\mathcal{P}_{r} \Lambda^{k}\left(\mathbb{R}^{n}\right)$, $\mathcal{J}_{r} \Lambda^{k}\left(\mathbb{R}^{n}\right)$, and $d \mathcal{J}_{r+1} \Lambda^{k-1}\left(\mathbb{R}^{n}\right)$ are contained in

$$
\mathcal{S}_{r} \Lambda^{k}(f)=\mathcal{P}_{r} \Lambda^{k}(f)+\mathcal{J}_{r} \Lambda^{k}(f)+d \mathcal{J}_{r+1} \Lambda^{k-1}(f) .
$$

For the $\mathcal{P}_{r} \Lambda^{k}\left(\mathbb{R}^{n}\right)$, this is evident, since $\operatorname{tr}_{f} \mathcal{P}_{r} \Lambda^{k}\left(\mathbb{R}^{n}\right) \subset \mathcal{P}_{r} \Lambda^{k}(f)$.

Next, we establish that $\operatorname{tr}_{f} d \mathcal{J}_{r+1} \Lambda^{k-1}\left(\mathbb{R}^{n}\right) \subset \mathcal{S}_{r} \Lambda^{k}(f)$. Indeed,

$$
\begin{aligned}
\operatorname{tr}_{f} d \mathcal{J}_{r+1} \Lambda^{k-1}\left(\mathbb{R}^{n}\right)=d \operatorname{tr}_{f} & \mathcal{J}_{r+1} \Lambda^{k-1}\left(\mathbb{R}^{n}\right) \\
& \subset d \operatorname{tr}_{f} \mathcal{S}_{r+1} \Lambda^{k-1}\left(\mathbb{R}^{n}\right) \subset d \mathcal{S}_{r+1} \Lambda^{k-1}(f) \subset \mathcal{S}_{r} \Lambda^{k}(f),
\end{aligned}
$$

where we have used, in turn, the commutativity of the trace with exterior differentiation, (17), the inductive hypothesis, and Theorem 3.3.

By Proposition 3.1, in order to show that $\operatorname{tr}_{f} \mathcal{J}_{r} \Lambda^{k}\left(\mathbb{R}^{n}\right) \subset \mathcal{S}_{r} \Lambda^{k}(f)$, and to complete the proof, it suffices to show that $\operatorname{tr}_{f} \kappa m \in \mathcal{S}_{r} \Lambda^{k}(f)$ whenever $m$ is a $(k+1)$-form monomial with $\operatorname{deg} m-\operatorname{ldeg} m \leq r-1$. We write $m$ as $x^{\alpha} d x_{\sigma}$, and consider separately the cases $1 \notin \sigma$ and $1 \in \sigma$.

Assuming $1 \notin \sigma$, let $p$ be the $(k+1)$-form monomial obtained restricting $m$ to $f$, i.e., by setting $x_{1}=c$ in the coefficient $x^{\alpha}$. Then $\operatorname{tr}_{f} \kappa m=\kappa p$. If $m$ is linear in $x_{1}$, then $\operatorname{deg} p=\operatorname{deg} m-1$ and $\operatorname{ldeg} p=\operatorname{ldeg} m-1$. Otherwise $\operatorname{deg} p \leq \operatorname{deg} m$ and $\operatorname{ldeg} p=\operatorname{ldeg} m$. In either event, $\operatorname{deg} p-\operatorname{ldeg} p \leq \operatorname{deg} m-\operatorname{ldeg} m \leq r-1$, so $\kappa p \in \mathcal{S}_{r} \Lambda^{k}(f)$ (again using Proposition 3.1).

Assuming, instead, that $1 \in \sigma$, we may write

$$
m=x^{\alpha} d x_{\sigma}=x_{1}^{\alpha_{1}} x^{\beta} d x_{1} \wedge d x_{\tau},
$$

where $\beta$ is a multi-index with $\beta_{1}=0$ and $\tau \in \Sigma(k)$ has $\tau_{1}>1$. Then

$$
p:=\operatorname{tr}_{f} \kappa m=c^{\alpha_{1}+1} x^{\beta} d x_{\tau}
$$

is a $k$-form monomial independent of $x_{1}$ with $\operatorname{deg} p \leq \operatorname{deg} m$ and $\operatorname{ldeg} p=\operatorname{ldeg} m$. We are trying to show that $p \in \mathcal{S}_{r} \Lambda^{k}(f)$. This is obvious if $\operatorname{deg} p \leq r$, so we may assume that $\operatorname{deg} p>r$. We shall show that both $\kappa d p$ and $d \kappa p$ belong to $\mathcal{S}_{r} \Lambda^{k}(f)$, which suffices by (9).

Now $\operatorname{deg} d p=\operatorname{deg} p-1 \leq \operatorname{deg} m-1$ and $\operatorname{ldeg} d p \geq \operatorname{ldeg} p-1=\operatorname{ldeg} m-1$, so $\operatorname{deg} d p-\operatorname{ldeg} d p \leq \operatorname{deg} m-\operatorname{ldeg} m \leq r-1$. Therefore $\kappa d p \in \mathcal{J}_{r} \Lambda^{k}(f) \subset \mathcal{S}_{r} \Lambda^{k}(f)$, as required.

Finally, we show that $\kappa p \in \mathcal{J}_{r+1} \Lambda^{k-1}(f)$, whence $d \kappa p \in \mathcal{S}_{r} \Lambda^{k}(f)$ as well. By Proposition 3.1 this holds, since $\operatorname{deg} p \geq r+1$ and $\operatorname{deg} p-\operatorname{ldeg} p \leq \operatorname{deg} m-\operatorname{ldeg} m \leq r$ (even $\leq r-1)$. This concludes the proof. 
Having defined the space $\mathcal{S}_{r} \Lambda^{k}\left(\mathbb{R}^{n}\right)$ of polynomial differential forms, we turn now to the definition of the associated finite element space on a cubical mesh. As usual the finite element space is defined element by element, by specifying a space of shape functions and a set of degrees of freedom on each cube $T$ in the mesh. (More generally the element $T$ may be a right rectangular prism, that is, the Cartesian product of $n$ closed intervals of positive finite length.) As shape functions on $T$ we use $\mathcal{S}_{r} \Lambda^{k}(T)$, the restriction of the above polynomial space to the cube. The degrees of freedom have a very simple expression. Writing $\Delta_{d}(T)$ for the set of $d$-dimensional faces of $T$, they are given by

$\mu \mapsto \int_{f} \operatorname{tr}_{f} \mu \wedge \nu, \quad \nu \in \mathcal{P}_{r-2(d-k)} \Lambda^{d-k}(f), f \in \Delta_{d}(T), k \leq d \leq \min (n,\lfloor r / 2\rfloor+k)$.

Since

$$
\operatorname{dim} \mathcal{P}_{r-2(d-k)} \Lambda^{d-k}(f)=\operatorname{dim} \mathcal{P}_{r-2(d-k)}(f) \times\left(\begin{array}{c}
d \\
d-k
\end{array}\right)=\left(\begin{array}{c}
r-d+2 k \\
d
\end{array}\right)\left(\begin{array}{c}
d \\
d-k
\end{array}\right)
$$

for $f$ a face of dimension $d$, and since there are $2^{n-d}\left(\begin{array}{l}n \\ d\end{array}\right) d$-dimensional faces of an $n$-cube, the number of degrees of freedom in (21) is given by

$$
\sum_{d=k}^{\min (n,\lfloor r / 2\rfloor+k)} 2^{n-d}\left(\begin{array}{l}
n \\
d
\end{array}\right)\left(\begin{array}{c}
r-d+2 k \\
d
\end{array}\right)\left(\begin{array}{l}
d \\
k
\end{array}\right) .
$$

We now turn to one of the main results of this paper, the proof that the degrees of freedom (21) are unisolvent for $\mathcal{S}_{r} \Lambda^{k}(T)$.

Theorem 3.6 (Unisolvence). Let $n, r \geq 1$ and $0 \leq k \leq n$, and let $T$ be a cube in $\mathbb{R}^{n}$. Then

(1) $\operatorname{dim} \mathcal{S}_{r} \Lambda^{k}(T)$ is given by (22).

(2) If $\mu \in \mathcal{S}_{r} \Lambda^{k}(T)$ and all the degrees of freedom in (21) vanish, then $\mu \equiv 0$.

Using the trace property, we will reduce the proof of (2) to the case where $\mu$ belongs to the space

$$
\stackrel{\circ}{\mathcal{S}}_{r} \Lambda^{k}(T):=\left\{\mu \in \mathcal{S}_{r} \Lambda^{k}(T) \mid \operatorname{tr}_{f} \mu=0 \text { on each face } f \in \Delta_{n-1}(T)\right\},
$$

the subspace with vanishing traces. This case is given in the following proposition.

Proposition 3.7. If $\mu \in \stackrel{\mathcal{S}}{r}_{r} \Lambda^{k}(T)$ and

$$
\int_{T} \mu \wedge \nu=0, \quad \nu \in \mathcal{P}_{r-2(n-k)} \Lambda^{n-k}(T),
$$

then $\mu$ vanishes.

We defer the proof of this proposition to Section 4. Now, assuming this result, we prove Theorem 3.6 .

Proof. We begin with the first statement of the theorem. Since $\mathcal{J}_{r} \Lambda^{k}(T)$ is in the range of $\kappa$, and since the homotopy formula implies that no nonzero differential form is in the range of both $\kappa$ and $d$, the sum on the left of (17) is direct. The homotopy formula implies as well that $d$ is injective on the range of $\kappa$. Therefore $\operatorname{dim} \mathcal{S}_{r} \Lambda^{k}(T)=\operatorname{dim} \mathcal{P}_{r} \Lambda^{k}(T)+\sum_{l \geq 1} \operatorname{dim} \kappa \mathcal{H}_{r+l-1, l} \Lambda^{k+1}(T)+\sum_{l \geq 1} \operatorname{dim} \kappa \mathcal{H}_{r+l, l} \Lambda^{k}(T)$. 
Applying Theorem 2.3 this becomes

$$
\begin{aligned}
\operatorname{dim} \mathcal{S}_{r} \Lambda^{k}(T)=\operatorname{dim} \mathcal{P}_{r} \Lambda^{k}(T)+\sum_{l \geq 1} \operatorname{dim} \mathcal{H}_{r+l, l} \Lambda^{k}(T) & \\
& =\operatorname{dim}\left[\mathcal{P}_{r} \Lambda^{k}(T)+\sum_{l \geq 1} \mathcal{H}_{r+l, l} \Lambda^{k}(T)\right] .
\end{aligned}
$$

The space in brackets is exactly the span of the $k$-form monomials $m$ with $\operatorname{deg} m-$ $\operatorname{ldeg} m \leq r$, and hence we need only count these monomials. This gives

$$
\operatorname{dim} \mathcal{S}_{r} \Lambda^{k}(T)=\left(\begin{array}{l}
n \\
k
\end{array}\right) \# A(r, k, n)
$$

where $\left(\begin{array}{l}n \\ k\end{array}\right)$ is the number of basic alternators and $A(r, n, k)$ is the set of monomials $p$ in $n$ variables which are linear in some number $l$ of the first $n-k$ variables, $x_{1}, \ldots, x_{n-k}$, with $\operatorname{deg} p-l \leq r$. Now we count the elements of $A(r, n, k)$. For any monomial $p$ in $n$ variables let $J \subset \mathbb{N}_{n-k}$ be the set of indices for which $x_{i}$ enters $p$ superlinearly, let $d \geq 0$ be the cardinality of $J$, and for $i \in \mathbb{N}_{n-k} \backslash J$, let $a_{i}=0$ or 1 according to whether $p$ is of degree 0 or 1 in $x_{i}$. Then

$$
p=\left(\prod_{j \in J} x_{j}^{2}\right) \times q \times\left(\prod_{i \in \mathbb{N}_{n-k} \backslash J} x_{i}^{a_{i}}\right),
$$

where $q$ is a monomial in the $d$ variables indexed by $J$ and the last $k$ variables. With $l$ the number of $a_{i}$ equal to 1 , we have $\operatorname{deg} p=2 d+\operatorname{deg} q+l$. Thus $\operatorname{deg} p-l \leq r$ if and only if $\operatorname{deg} q \leq r-2 d$. Thus we may uniquely specify an element of $A(r, k, n)$ by choosing $d \geq 0$, choosing the set $J$ consisting of $d$ of the $n-k$ variables (for which there are $\left(\begin{array}{c}n-k \\ d\end{array}\right)$ possibilities), choosing the monomial $q$ of degree at most $r-2 d$ in the $d+k$ variables $\left(\left(\begin{array}{c}r-d+k \\ d+k\end{array}\right)\right.$ possibilities $)$, and choosing the exponent $a_{i}$ to be either 0 or 1 for the $n-k-d$ remaining indices $\left(2^{n-k-d}\right.$ possibilities). Thus

$$
\begin{aligned}
\# A(r, k, n) & =\sum_{d=0}^{\min (n-k,\lfloor r / 2\rfloor)} 2^{n-k-d}\left(\begin{array}{c}
n-k \\
d
\end{array}\right)\left(\begin{array}{c}
r-d+k \\
d+k
\end{array}\right) \\
& =\sum_{d=k}^{\min (n,\lfloor r / 2\rfloor+k)} 2^{n-d}\left(\begin{array}{c}
n-k \\
d-k
\end{array}\right)\left(\begin{array}{c}
r-d+2 k \\
d
\end{array}\right),
\end{aligned}
$$

where the second sum comes from a change of the summation index $(d \rightarrow d-k)$. Substituting (25) into (24) and using the binomial identity

$$
\left(\begin{array}{l}
n \\
k
\end{array}\right)\left(\begin{array}{l}
n-k \\
d-k
\end{array}\right)=\left(\begin{array}{l}
d \\
k
\end{array}\right)\left(\begin{array}{l}
n \\
d
\end{array}\right)
$$

we conclude that

$$
\operatorname{dim} \mathcal{S}_{r} \Lambda^{k}(T)=\sum_{d=k}^{\min (n,\lfloor r / 2\rfloor+k)} 2^{n-d}\left(\begin{array}{l}
n \\
d
\end{array}\right)\left(\begin{array}{c}
r-d+2 k \\
d
\end{array}\right)\left(\begin{array}{l}
d \\
k
\end{array}\right) .
$$

This completes the proof of the dimension formula for $\mathcal{S}_{r} \Lambda^{k}\left(\mathbb{R}^{n}\right)$.

The proof of unisolvence is easily completed based on the trace property and Proposition 3.7. We use induction on the dimension $n$, the one-dimensional case being trivial. Suppose $\mu \in \mathcal{S}_{r} \Lambda^{k}(T)$ and all its degrees of freedom vanish. For any face $f$ of dimension $n-1, \operatorname{tr}_{f} \mu \in S_{r} \Lambda^{k}(f)$ and all the degrees of freedom for it 
vanish. By induction $\operatorname{tr}_{f} \mu \equiv 0$ on $f$. This implies that $\mu \in \stackrel{\mathcal{S}}{r}_{r} \Lambda^{k}(T)$, and we invoke Proposition 3.7 to conclude that $\mu$ vanishes identically.

We remark that, as a corollary of unisolvence, we may strengthen the result of Theorem 3.5 to equality

$$
\operatorname{tr}_{f} \mathcal{S}_{r} \Lambda^{k}\left(\mathbb{R}^{n}\right)=\mathcal{S}_{r} \Lambda^{k}(f)
$$

Indeed, let $T$ be a cube with one face contained in the hyperplane $f$. Then $T \cap f$ is an $(n-1)$-dimensional cube and any $\nu \in \mathcal{S}_{r} \Lambda^{k}(f)$ is uniquely determined by the degrees of freedom for the $\mathcal{S}_{r} \Lambda^{k}(T \cap f)$. Now we may determine an element $\mu \in \mathcal{S}_{r} \Lambda^{k}\left(\mathbb{R}^{n}\right)$ by assigning the degrees of freedom for the space $\mathcal{S}_{r} \Lambda^{k}(T)$ arbitrarily. In particular, we may choose the values of those degrees of freedom associated to the face $T \cap f$ and its subfaces to be the same as those for $\nu$. Then $\operatorname{tr}_{f} \mu \in \mathcal{S}_{r} \Lambda^{k}(f)$ (by Theorem 3.5), and $\operatorname{tr}_{f} \mu$ and $\nu$ have identical degrees of freedom, and so they are equal (by Theorem 3.6).

With the definition of the spaces complete, we use the subcomplex property, Theorem 3.3 to define a subcomplex of the de Rham complex on the cube

$$
\mathbb{R} \stackrel{\subset}{\longrightarrow} \mathcal{S}_{r} \Lambda^{0}(T) \stackrel{d}{\longrightarrow} \mathcal{S}_{r-1} \Lambda^{1}(T) \stackrel{d}{\longrightarrow} \cdots \stackrel{d}{\longrightarrow} \mathcal{S}_{r-n} \Lambda^{n}(T) \longrightarrow 0 .
$$

To show that this complex is exact, we define the canonical projection

$$
\pi_{r}^{k}: C \Lambda^{k}(T) \rightarrow \mathcal{S}_{r} \Lambda^{k}(T),
$$

associated to the unisolvent degrees of freedom. That is, $\pi_{r}^{k} \mu \in \mathcal{S}_{r} \Lambda^{k}(T)$ is determined by the equations

$$
\begin{array}{r}
\int_{f} \operatorname{tr}_{f}\left(\pi_{r}^{k} \mu\right) \wedge \nu=\int_{f} \operatorname{tr}_{f} \mu \wedge \nu, \quad \nu \in \mathcal{P}_{r-2(d-k)} \Lambda^{d-k}(f), f \in \Delta_{d}(T), \\
k \leq d \leq \min (n,\lfloor r / 2\rfloor+k) .
\end{array}
$$

Then, the following diagram commutes:

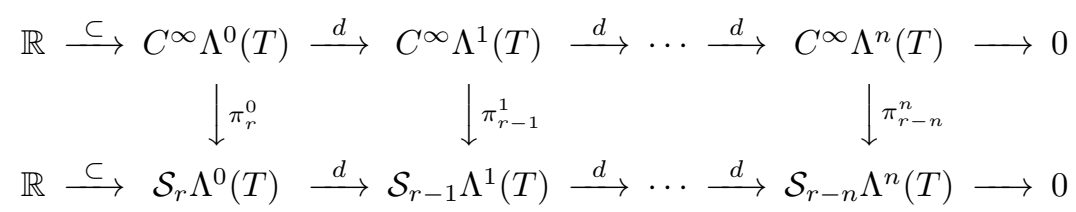

The proof of commutativity is based on two basic properties of differential forms:

(1) the commutativity of trace and exterior differentiation, $\operatorname{tr}_{f} d \omega=d_{f} \operatorname{tr}_{f} \omega$, and

(2) integration by parts, which for differential forms can be written as

$$
\int_{\Omega} d \omega \wedge \eta=(-1)^{k-1} \int_{\Omega} \omega \wedge d \eta+\int_{\partial \Omega} \operatorname{tr}_{\partial \Omega} \omega \wedge \operatorname{tr}_{\partial \Omega} \eta
$$

for a $k$-form $\omega$ and an $(n-k-1)$-form $\eta$ on an $n$-dimensional domain $\Omega$. See [4. Lemma 4.24] for the same argument applied to simplicial elements. Since the top row of the diagram, the de Rham complex on the cube, is exact, the commutativity of the diagram implies that the bottom row is exact as well.

Having defined the finite element space $\mathcal{S}_{r} \Lambda^{k}(T)$ on a single cube $T$ and established its properties, the space $\mathcal{S}_{r} \Lambda^{k}\left(\mathcal{T}_{h}\right)$ associated to a cubical mesh $\mathcal{T}_{h}$ is defined through the usual finite element assembly. In view of the unisolvence result Theorem 3.6 and the trace result (27), the degrees of freedom associated to a face of the cube and its subfaces determine the trace of the finite element differential form on the face. It follows that $\mathcal{S}_{r} \Lambda^{k}\left(\mathcal{T}_{h}\right) \subset H \Lambda^{k}(\Omega)$ (see [4, Section 5.1]). 


\section{Unisolvence OVER The SPACE With VANishing TRACES}

We conclude the paper with the proof of Proposition 3.7, which is based on the following lemma.

Lemma 4.1. Suppose that $\eta=\sum_{\sigma \in \Sigma(k)} \eta_{\sigma} d x_{\sigma}$ where, for each $\sigma \in \Sigma(k), \eta_{\sigma}$ is a homogeneous polynomial which is superlinear in all the $\sigma^{*}$ variables. Further suppose that $\operatorname{ldeg} \kappa \eta \geq 1$ and $\operatorname{ldeg} \kappa d \eta \geq 1$. Then $\eta=0$.

Proof of Lemma 4.1. By (8) we have $\kappa d \eta=\sum_{\sigma \in \Sigma(k)} \mu_{\sigma} d x_{\sigma}$, where

$$
\mu_{\sigma}=\sum_{q \in \sigma^{*}}\left[x_{q} \partial_{q} \eta_{\sigma}+\sum_{p \in \sigma} \epsilon(q, p, \sigma) x_{q} \partial_{p} \eta_{\sigma+q-p}\right] .
$$

Since ldeg $\kappa d \eta \geq 1$, each monomial of the polynomial $\mu_{\sigma}$ is linear in at least one $\sigma^{*}$ variable.

For any $\sigma \in \Sigma(k)$ and any subset $\tau$ of $\sigma$, let $S_{\sigma}^{\tau}$ be the span of the (ordinary, 0 -form) monomials which are independent of the $\tau$ variables but depend on all of the other $\sigma$ variables, and let $S_{\sigma}^{*}$ denote the span of the monomials which are superlinear in all the $\sigma^{*}$ variables. Denote by $P_{\sigma}^{\tau}$ the projection onto $S_{\sigma}^{\tau}$. That is, if $p=\sum p_{m} m$ where the sum is over all monomials $m$ and the coefficients $p_{m}$ are real numbers, all but finitely many zero, then $P_{\sigma}^{\tau} p:=\sum_{m \in S_{\sigma}^{\tau}} p_{m} m$. Similarly, we denote by $Q_{\sigma}^{\tau}$ the projection onto $S_{\sigma}^{\tau} \cap S_{\sigma}^{*}$. We now calculate the result of applying $Q_{\sigma}^{\tau}$ to both sides of (28). First we note that

$$
Q_{\sigma}^{\tau} \mu_{\sigma}=0
$$

since every monomial of $\mu_{\sigma}$ is linear in at least one $\sigma^{*}$ variable, and so none of them belong to $S_{\sigma}^{*}$. Next, for each $q \in \sigma^{*}$,

$$
Q_{\sigma}^{\tau}\left(x_{q} \partial_{q} \eta_{\sigma}\right)=P_{\sigma}^{\tau}\left(x_{q} \partial_{q} \eta_{\sigma}\right)=x_{q} \partial_{q}\left(P_{\sigma}^{\tau} \eta_{\sigma}\right),
$$

with the first inequality holding since each monomial of $\eta_{\sigma}$, and therefore also of $x_{q} \partial_{q} \eta_{\sigma}$, is superlinear in all of the $\sigma$ variables. Now we determine the action of $Q_{\sigma}^{\tau}$ on the terms of the second sum on the right-hand side of (28). For any $q \in \sigma^{*}$ and $p \in \sigma$, we claim that

$$
Q_{\sigma}^{\tau}\left(x_{q} \partial_{p} \eta_{\sigma+q-p}\right)= \begin{cases}0, & p \in \tau, \\ x_{q} \partial_{p}\left(P_{\sigma+q-p}^{\tau} \eta_{\sigma+q-p}\right), & p \in \sigma \backslash \tau .\end{cases}
$$

Indeed, $\eta_{\sigma+q-p}$ is superlinear in $x_{p}$, so every monomial of $x_{q} \partial_{p} \eta_{\sigma+q-p}$ depends on $x_{p}$. This implies that the projection is 0 in the case $p \in \tau$. In case $p \in \sigma \backslash \tau$, we write $x_{q} \partial_{p} \eta_{\sigma+q-p}=\sum x_{q} \partial_{p} m$, where the sum is over the monomials $m$ of $\eta_{\sigma+q-p}$. Since neither $p$ or $q$ belongs to $\tau$, the monomial $m^{\prime}:=x_{q} \partial_{p} m$ is independent of the $\tau$ variables if and only if the same is true of the monomial $m$, and, since $m^{\prime}$ always depends on $x_{p}$, it depends on all of the $\sigma \backslash \tau$ variables if and only if $m$ depends on all of the $(\sigma-p) \backslash \tau$ variables. Further, $m^{\prime}$ is always superlinear in all of the $\sigma^{*}$ variables except possibly $x_{q}$, and it is superlinear in $x_{q}$ if and only if $m$ depends on $x_{q}$. In short, $m^{\prime}$ belongs to $S_{\sigma}^{\tau} \cap S_{\sigma}^{*}$ if and only if $m$ belongs to $S_{\sigma+q-p}^{\tau}$. This completes the verification of (31). Thus the application of $Q_{\sigma}^{\tau}$ to (28) gives, in light of (29), (30), and (31), that

$$
\sum_{q \in \sigma^{*}}\left[x_{q} \partial_{q}\left(P_{\sigma}^{\tau} \eta_{\sigma}\right)+\sum_{p \in \sigma \backslash \tau} \epsilon(q, p, \sigma) x_{q} \partial_{p}\left(P_{\sigma+q-p}^{\tau} \eta_{\sigma+q-p}\right)\right]=0 .
$$


We now claim that, for any $\sigma \in \Sigma(k)$ and $\tau \subset \sigma$, that

$$
\sum_{q \in \sigma^{*}} \sum_{p \in \sigma \backslash \tau} \epsilon(q, p, \sigma) x_{q} \partial_{p}\left(P_{\sigma+q-p}^{\tau} \eta_{\sigma+q-p}\right)=\left(c+\sum_{i \in \sigma \backslash \tau} x_{i} \partial_{i}\right)\left(P_{\sigma}^{\tau} \eta_{\sigma}\right),
$$

where $c=\#(\sigma \backslash \tau)$. Assuming this, we have from (32),

$0=\left(c+\sum_{i \in \sigma \backslash \tau} x_{i} \partial_{i}+\sum_{q \in \sigma^{*}} x_{q} \partial_{q}\right)\left(P_{\sigma}^{\tau} \eta_{\sigma}\right)=\left(c+\sum_{i \in \tau^{*}} x_{i} \partial_{i}\right)\left(P_{\sigma}^{\tau} \eta_{\sigma}\right)=(c+r+1)\left(P_{\sigma}^{\tau} \eta_{\sigma}\right)$,

where we have used Euler's formula for homogeneous polynomials (i.e., the homotopy formula for 0 -forms) in the last step. Thus, $P_{\sigma}^{\tau} \eta_{\sigma}$ vanishes, and so $\eta_{\sigma}=$ $\sum_{\tau \subset \sigma} P_{\sigma}^{\tau} \eta_{\sigma}$ also vanishes. Since $\sigma \in \Sigma(k)$ is arbitrary, this implies that $\eta=0$.

Thus it remains only to prove (33). By the first formula of Lemma 4, $\kappa \eta=$ $\sum_{\zeta \in \Sigma(k-1)} \omega_{\zeta} d x_{\zeta}$ where

$$
\omega_{\zeta}=\sum_{q \in \zeta^{*}} \epsilon(q, \zeta) x_{q} \eta_{\zeta+q}
$$

Now let $\zeta \in \Sigma(k-1)$ and $\tau \subset \zeta$, and denote by $R_{\zeta}^{\tau}$ the projection onto $S_{\zeta}^{\tau} \cap S_{\zeta}^{*}$ (the span of monomials which depend on all the $\zeta$ variables except the $\tau$ variables and which are superlinear in the $\zeta^{*}$ variables). By the hypothesis that ldeg $\kappa \eta \geq 1$,

$$
R_{\zeta}^{\tau} \omega_{\zeta}=0 \text {. }
$$

Next we compute $R_{\zeta}^{\tau}\left(x_{q} \eta_{\zeta+q}\right)$ for $q \in \zeta^{*}$. If $m$ is a monomial of $\eta_{\zeta+q}$, then $m^{\prime}=$ $x_{q} \eta_{\zeta+q}$ depends on all the $\zeta$ variables except for the $\tau$ variables if and only if the same is true of $m$. Moreover, $m^{\prime}$ is superlinear in all the $\zeta^{*}$ variables if and only if $m$ depends on $x_{q}$ (since $m$ is superlinear in all the $\zeta^{*}$ variables with the possible exception of $x_{q}$ ). Thus,

$$
R_{\zeta}^{\tau}\left(x_{q} \eta_{\zeta+q}\right)=x_{q} P_{\zeta+q}^{\tau} \eta_{\zeta+q}
$$

Combining the last three displayed equations, we obtain

$$
\sum_{q \in \zeta^{*}} \epsilon(q, \zeta) x_{q} P_{\zeta+q}^{\tau} \eta_{\zeta+q}=0 .
$$

Now choose some $p \in \zeta^{*}$ and differentiate this equation with respect to $x_{p}$ to get

$$
\epsilon(p, \zeta) \partial_{p}\left(x_{p} P_{\zeta+p}^{\tau} \eta_{\zeta+p}\right)+\sum_{q \in \zeta^{*}-p} \epsilon(q, \zeta) x_{q} \partial_{p}\left(P_{\zeta+q}^{\tau} \eta_{\zeta+q}\right)=0,
$$

or, after rearranging,

$$
P_{\zeta+p}^{\tau} \eta_{\zeta+p}+x_{p} \partial_{p}\left(P_{\zeta+p}^{\tau} \eta_{\zeta+p}\right)=-\sum_{q \in \zeta^{*}-p} \epsilon(p, \zeta) \epsilon(q, \zeta) x_{q} \partial_{p}\left(P_{\zeta+q}^{\tau} \eta_{\zeta+q}\right) .
$$

For any $\sigma \in \Sigma(k)$ and any $p \in \sigma$ set $\zeta=\sigma-p \in \Sigma(k-1)$. Then we can rewrite (34) in terms of $\sigma$ as

$$
P_{\sigma}^{\tau} \eta_{\sigma}+x_{p} \partial_{p}\left(P_{\sigma}^{\tau} \eta_{\sigma}\right)=-\sum_{q \in \sigma^{*}} \epsilon(p, \sigma-p) \epsilon(q, \sigma-p) x_{q} \partial_{p}\left(P_{\sigma+q-p}^{\tau} \eta_{\sigma+q-p}\right) .
$$

Finally, taking any $\tau \subset \sigma \in \Sigma(k)$, we sum over $p \in \sigma \backslash \tau$ to obtain $c P_{\sigma}^{\tau} \eta_{\sigma}+\sum_{p \in \sigma \backslash \tau} x_{p} \partial_{p}\left(P_{\sigma}^{\tau} \eta_{\sigma}\right)=-\sum_{p \in \sigma \backslash \tau} \sum_{q \in \sigma^{*}} \epsilon(p, \sigma-p) \epsilon(q, \sigma-p) x_{q} \partial_{p}\left(P_{\sigma+q-p}^{\tau} \eta_{\sigma+q-p}\right)$, where $c=\#(\sigma \backslash \tau)$. In light of (3), this establishes (33), and so completes the proof of the lemma. 
Finally, we give the proof of Proposition 3.7.

Proof. By dilating and translating, it suffices to prove the result when $T=I^{n}$ with $I=[-1,1]$. Let

$$
\mu=\sum_{\sigma \in \Sigma(k)} \mu_{\sigma} d x_{\sigma}
$$

be a polynomial differential form on the cube $I^{n}$. Then $\operatorname{tr}_{f} \mu$ vanishes on the faces $x_{i}= \pm 1$ if and only if for each $\sigma$ such that $i \notin \sigma, 1-x_{i}^{2}$ divides $\mu_{\sigma}$. Now suppose that $\mu \in \stackrel{\circ}{\mathcal{S}}_{r} \Lambda^{k}\left(I^{n}\right)$, so that $\operatorname{tr}_{f} \mu$ vanishes on all the faces of the cube. It follows that

$$
\mu_{\sigma}=\tilde{\mu}_{\sigma} \prod_{i \in \sigma^{*}}\left(1-x_{i}^{2}\right)
$$

for some polynomial $\tilde{\mu}_{\sigma}$. The monomial expansion of $\mu$ then contains the form monomial $m_{\sigma} \prod_{i \in \sigma^{*}} x_{i}^{2} d x_{\sigma}$, where $m_{\sigma}$ is any monomial of highest degree of $\tilde{\mu}_{\sigma}$. The linear degree of this form monomial is 0 , so, by the degree property (20), its degree is at most $r+1$.

Having established that $\mu$ is of degree at most $r+1$, let $\eta$ be its homogeneous part of degree $r+1$. We have $\operatorname{ldeg} \eta=0$. Now we may match terms in the definition (17) of $\mathcal{S}_{r} \Lambda^{k}(\Omega)$ to obtain that $\eta=\kappa v+d \kappa \omega$, for some $v \in \mathcal{H}_{r, 1} \Lambda^{k+1}\left(I^{n}\right)$ and $\omega \in \mathcal{H}_{r+1,1} \Lambda^{k}\left(I^{n}\right)$. Therefore, $\operatorname{ldeg} \kappa \eta=\operatorname{ldeg} \kappa d \kappa \omega \geq \operatorname{ldeg} \omega \geq 1$ and $\operatorname{ldeg} \kappa d \eta=$ $\operatorname{ldeg} \kappa d \kappa v \geq \operatorname{ldeg} v \geq 1$, where we have used Lemma 2.2

By Lemma 4.1, $\eta=0$, hence the monomial of highest order in the expansion of $\mu_{\sigma}$ is of degree at most $r$. It follows that $\tilde{\mu}_{\sigma}$ is of degree $r-2(n-k)$ for each $\sigma \in \Sigma(k)$. We can then choose the test function $\nu=\sum_{\sigma \in \Sigma(k)}(-1)^{\operatorname{sgn}\left(\sigma, \sigma^{*}\right)} \tilde{\mu}_{\sigma} d x_{\sigma^{*}}$ in (23) to conclude that $\mu$ vanishes.

\section{REFERENCES}

[1] Scot Adams and Victor Reiner, private communication.

[2] Douglas N. Arnold and Gerard Awanou, The serendipity family of finite elements, Found. Comput. Math. 11 (2011), no. 3, 337-344, DOI 10.1007/s10208-011-9087-3. MR2794906 (2012i:65249)

[3] Douglas N. Arnold, Daniele Boffi, and Francesca Bonizzoni, Tensor product finite element differential forms and their approximation properties, preprint 2012, arXiv: 1212.6559 [math.NA].

[4] Douglas N. Arnold, Richard S. Falk, and Ragnar Winther, Finite element exterior calculus, homological techniques, and applications, Acta Numer. 15 (2006), 1-155, DOI 10.1017/S0962492906210018. MR2269741 (2007j:58002)

[5] Douglas N. Arnold, Richard S. Falk, and Ragnar Winther, Finite element exterior calculus: from Hodge theory to numerical stability, Bulletin of the American Mathematical Society 42 (2010), no. 2, 281-354. MR2594630 (2011f:58005)

[6] Franco Brezzi, Jim Douglas Jr., Ricardo Durán, and Michel Fortin, Mixed finite elements for second order elliptic problems in three variables, Numer. Math. 51 (1987), no. 2, 237-250, DOI 10.1007/BF01396752. MR890035 (88f:65190)

[7] Franco Brezzi, Jim Douglas Jr., and L. D. Marini, Two families of mixed finite elements for second order elliptic problems, Numer. Math. 47 (1985), no. 2, 217-235, DOI 10.1007/BF01389710. MR799685 (87g:65133)

[8] Runchang Lin and Zhimin Zhang, Natural superconvergence points in three-dimensional finite elements, SIAM J. Numer. Anal. 46 (2008), no. 3, 1281-1297, DOI 10.1137/070681168. MR.2390994 (2009a:65321)

[9] Jean-Claude Nédélec, Mixed finite elements in $\mathbf{R}^{3}$, Numerische Mathematik 35 (1980), 315241. 
[10] J.-C. Nédélec, A new family of mixed finite elements in $\mathbf{R}^{3}$, Numer. Math. 50 (1986), no. 1, 57-81, DOI 10.1007/BF01389668. MR864305 (88e:65145)

[11] Pierre-Arnaud Raviart and Jean-Marie Thomas, A mixed finite element method for 2nd order elliptic problems, Mathematical aspects of finite element methods (Proc. Conf., Consiglio Naz. delle Ricerche (C.N.R.), Rome, 1975), Vol. 606 of Lecture Notes in Mathematics (Berlin), Springer, 1977, pp. 292-315. MR 483555 (58 \#3547)

[12] Barna Szabó and Ivo Babuška, Finite element analysis, A Wiley-Interscience Publication, John Wiley \& Sons Inc., New York, 1991. MR1164869 (93f:73001)

Department of Mathematics, University of Minnesota, Minneapolis, Minnesota 55455

E-mail address: arnold@umn.edu

Department of Mathematics, Statistics, and Computer Science, M/C 249, University of Illinois at Chicago, Chicago, Illinois 60607-7045

E-mail address: awanou@uic.edu 